\title{
Direct Measurement of Propagation Constant in Optical Waveguides by Heterodyne Scattering Detection (HSD) Method
}

\author{
Yasuo Kokubun, Yutaka Kai, and Hideya Johkawa
}

\begin{abstract}
A new measurement method for the propagation constant of optical waveguides, which can measure the phase difference of two distant points nondestructively, was proposed and demonstrated. A super-fine fiber probe for the photon STM is used to pick up the scattered light from an optical waveguide, and the collected light is detected using a heterodyne technique. The phase difference between two points is measured from the phase change of the electrical signal of heterodyne detection as the fiber probe traces the waveguide pattern. The phase difference between $100 \mu \mathrm{m}$ distant points in a three-dimensional ARROW was successfully measured and the error was evaluated to be $0.25 \%$ by comparison with the theoretical value.
\end{abstract}

\section{INTRODUCTION}

A MEASUREMENT technique for optical waveguides and waveguide type devices is indispensable for developing photonic integrated circuits (PIC's). One of most important items is the propagation loss including scattering, radiation and coupling losses, and several measurement methods such as the prism coupling method, the TV camera observation method [1] and some reflectometry methods [2]-[5] have been proposed. Another important item is the phase difference between two points along the waveguide pattern, or the propagation constant in the normalized form, because the phase difference between two points plays an important role in some lightwave circuits utilizing interference, such as a Mach-Zehnder interferometer and waveguide grating multi/demultiplexers. However, there have been reported no propagation constant measurement method which can measure the phase difference of two arbitrary distant points in a waveguide nondestructively and precisely. In this letter, a nondestructive measurement method for the propagation constant of a waveguide is proposed and demonstrated.

As a nondestructive measurement method for propagation loss, we have proposed and demonstrated the heterodyne scattering detection (HSD for short) method [6], [7]. In this method, a laser beam is divided into two arms, and one is guided in a waveguide. The scattered light is collected by a fiber probe and is mixed with the other arm of the laser beam, the frequency of which is shifted using a phase modulator. Thus the weak scattered light is detected by a heterodyne method. The propagation loss is measured by scanning the fiber probe along

Manuscript received June 13, 1995; revised July 13, 1995.

The authors are with Yokohama National University, Division of Electrical and Computer Engineering, 156 Tokiwadai, Hodogayaku, Yokohama, 240 Japan.

IEEE Log Number 9415366. the waveguide pattern. In contrast to reflectometry methods, the HSD method is applicable to a complex waveguide pattern including branching and mixing waveguides, because each arm of the branch can be traced independently by scanning the fiber probe on the objective arm. However, the spatial resolution was limited to be several microns due to the tip diameter of the fiber probe, although this value is better than those of reflectometry methods. In contrast to these, super-high resolution diagnostics of an optical waveguide using a photon scanning tunneling microscope (photon STM) was recently proposed and a high spatial resolution of $500 \mathrm{~nm}$ was demonstrated [8].

On the other hand, the HSD method can measure the phase change of collected light resulting from the shift of the fiber probe, because the phase change of the electrical heterodyne signal is equal to the phase change of the collected light. To realize this, however, a high spatial resolution of less than at least a quarter wavelength is needed for both the tip diameter of the fiber probe and the mechanical manipulator. The former requirement can be achieved by introducing a super-fine fiber probe for the photon STM [9]. The latter has already been satisfied in our measurement system, because the manipulator of our system (Nanomover made by MELLES GRIOT) has a spatial resolution of $50 \mathrm{~nm}$ and a reproducible resolution of $100 \mathrm{~nm}$. Therefore, we first prepared a super-fine fiber probe and then tried to measure the phase difference of two distant points along a waveguide. The propagation constant can be calculated from the phase change normalized by the distance.

\section{Measurement SetuP and Principle}

The measurement setup of the HSD method is shown in Fig. 1. Coherent light from an LD is divided into two arms by a fiber coupler, and one is incident on a sample waveguide. A narrow line-width DFB-LD emitting at $1.558 \mu \mathrm{m}$ wavelength with an isolator was used as a light source. The scattered light from the surface of the waveguide is picked up by a fiber probe. The other arm is guided in the fiber as a reference light signal and the optical frequency is shifted by $30-75 \mathrm{~Hz}$ by applying a saw-shaped voltage pattern to the phase modulator. The scattered light from the surface of waveguide is mixed with the reference light in the fiber coupler. The mixed light is detected by a photodiode, and the scattered light is detected by a heterodyne technique using a lock-in amplifier. The minimum detectable power was approximately $7 \mathrm{pW}$, when the output power of the LD was $3.4 \mathrm{~mW}$. All the fiber circuits are 


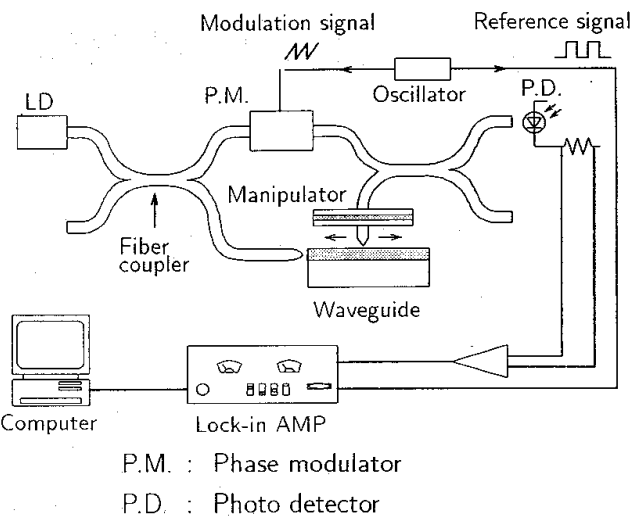

Fig. 1. Measurement setup of heterodyne scattering detection (HSD) method.

made of polarization maintaining fibers to stabilize the state of polarization. By moving the position of the fiber probe along the surface of the waveguide, we can measure the propagation loss and the position and intensity of a scattering center in an optical waveguide. This method can also measure the phase shift of the guided light against the movement of the fiber probe from the phase shift of the heterodyne signal.

The fiber probe was sharpened by wet chemical etching using the same etchant as was used for the fiber probe of the photon STM [9], and was coated with about $50 \mathrm{~nm}$ of chromium to avoid the noise resulting from the scattered light at the side surface of the fiber probe. Then the chromium on the tip was eliminated by a wet etching, while monitoring the radiation of a $\mathrm{He}-\mathrm{Ne}$ laser light from the tip. The tip diameter was less than $250 \mathrm{~nm}$, and typically about $150 \mathrm{~nm}$.

The measurement principle for the propagation constant of the guided light is shown in Fig. 2. When the position of the fiber probe is $z_{1}$, where $z_{1}$ is the distance from the input end to the objective point, the phase $\phi\left(z_{1}\right)$ of the heterodyne signal from the lock-in amplifier is given by the following equation

$$
\phi\left(z_{1}\right)=\beta \cdot z_{1}-\varphi\left(t_{1}\right)
$$

where $\beta$ is the propagation constant of the guided light in the optical waveguide, $\varphi\left(t_{1}\right)$ is the phase difference between the reference light and the scattered light, and $t_{1}$ is the time when the light is detected. $\varphi\left(t_{1}\right)$ changes slowly due to the vibration of the fibers and the change of temperature. When the position of the probe fiber is moved to $z_{2}$ at time $t_{2}$, the phase $\phi\left(z_{2}\right)$ of the heterodyne signal is given by

$$
\phi\left(z_{2}\right)=\beta \cdot z_{2}-\varphi\left(t_{2}\right) .
$$

Thus the phase difference $\Delta \phi$ between two points $z_{1}$ and $z_{2}$ is given by

$$
\begin{aligned}
\Delta \phi & =\phi\left(z_{2}\right)-\phi\left(z_{1}\right) \\
& =\beta\left(z_{2}-z_{1}\right)-\varphi\left(t_{2}\right)+\varphi\left(t_{1}\right) .
\end{aligned}
$$

If the ambient temperature and vibration of the measurement setup are stabilized, we can calculate the propagation constant $\beta$ from (3) as

$$
\beta \simeq \frac{\phi\left(z_{2}\right)-\phi\left(z_{1}\right)}{z_{2}-z_{1}}=\frac{\Delta \phi}{\Delta z}
$$

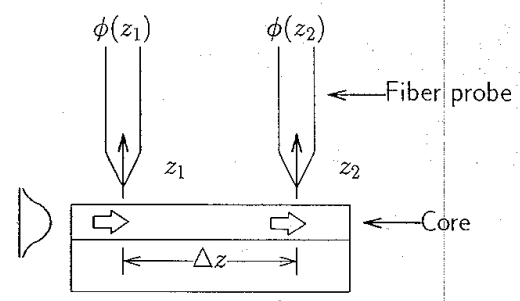

Fig. 2. Principle of measurement of phase difference between two points. $\phi\left(z_{1}\right)$ and $\phi\left(z_{2}\right)$ are the phase of scattered light at the positions $z_{1}$ and $z_{2}$, respectively.

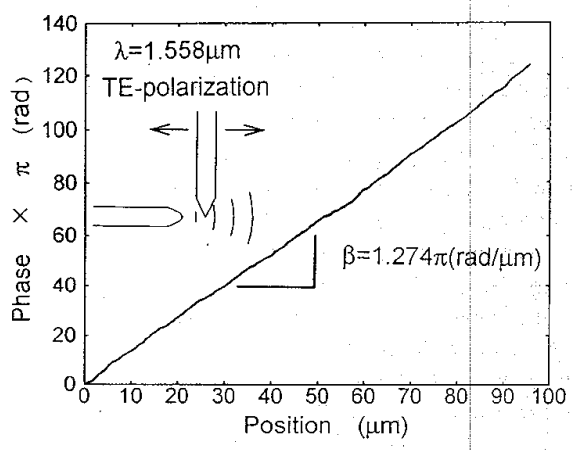

Fig. 3. Measured result of phase change of a spatial beam along the opitcal axis emitted from a fiber. The theoretical value of the propagation constant is $1.283 \pi(\mathrm{rad} / \mu \mathrm{m})$.

To improve the accuracy, the number of sampled points was increased to one thousand with an interval of $100 \mathrm{~nm}$, and the phase difference $\Delta \phi$ was accumulated beyond $2 \pi$. The propagation constant was calculated from the slope of the straight line relation between the phase $\phi(z)$ and the position $z$, which was approximated by a least squares fit.

In contrast to a PZT manipulator used in the photon STM, a mechanical manipulator can trace longer than several hundred microns ( $25 \mathrm{~mm}$ in our system). This long distance tracing can increase the number of sampled points and thus reduces the error due to the phase ambiguity $\varphi(t)$.

\section{EXPERIMENTS}

To confirm the accuracy of this method, we measured the propagation constant of the optical beam radiated from a single-mode fiber. The measured result of the propagation constant of the optical beam is shown in Fig. 3. Since the interval between adjacent points is $100 \mathrm{~nm}$, more than 900 points are involved in this measured result. The propagation constant was calculated to be $1.274 \pi(\mathrm{rad} / \mu \mathrm{m})$ from the least squares fit. Since the theoretical value is $1.283 \pi(\mathrm{rad} / \mu \mathrm{m})$, the error is evaluated to be less than $0.7 \%$.

In this measurement, the fiber probe was not coated with chromium. Although the core diameter of the fiber probe is greater than the wavelength, the light coupling from the optical beam to the fiber probe is considered to occur only at the fine tip of the fiber probe, because the phase shift was successfully measured. The fine tip of the fiber probe seems to act as the scattering center.

Next, we measured the propagation constant of the TE fundamental mode of a stripe lateral confinement (SLC) 


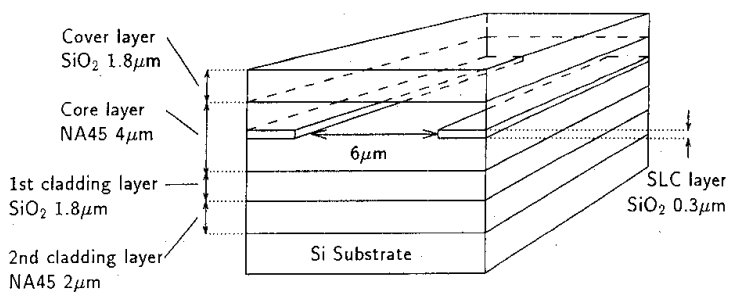

Fig. 4. Structure of ARROW-B waveguide used in the measurement of propagation constant. The width and thickness of core are $6 \mu \mathrm{m}$ and $4 \mu \mathrm{m}$, respectively. NA45 glass has the same composition as Corning 7059 glass. The refractive indices of $\mathrm{NA} 45$ glass and $\mathrm{SiO}_{2}$ are 1.55 and 1.467 , respectively.

ARROW-B waveguide as shown in Fig. 4. The distance between the surface of the waveguide and the fiber probe was kept to be about $1 \mu \mathrm{m}$ or less. The measured result of the phase change versus the tracing distance over $100 \mu \mathrm{m}$ is shown in Fig. 5. 1000 points are involved in this measured result. The propagation constant was calculated to be $1.959 \pi(\mathrm{rad} / \mu \mathrm{m})$ from the slope of the straight line which was approximated by a least squares fit. Since the theoretical value calculated from the waveguide structure is $1.9638 \pi(\mathrm{rad} / \mu \mathrm{m})$, the measurement error is evaluated to be less than $0.25 \%$.

\section{CONCLUSION}

In summary, we successfully realized the direct measurement of the propagation constant of an optical waveguide by the HSD method. To our knowledge, this is the first report of the direct, nondestructive measurement of a propagation constant of optical waveguide using a fiber probe. By combining the intensity measurement of scattered light with this phase measurement, the HSD method will provide us with a very strong tool for the diagnostics of waveguides and waveguide-type devices.

\section{ACKNOWLEDGMENT}

The authors express their sincere thanks to Prof. M. Ohtsu and Dr. Y. Toda of Tokyo Institute of Technology for teaching us the fabrication of the super-fine fiber probe. The authors also acknowledge Dr. M. Maeda of Hitachi Co. Ltd. for supplying an LD and Drs. S. Miyamoto and T. Watanabe of Fujikura Co. Ltd. for supplying fibers.

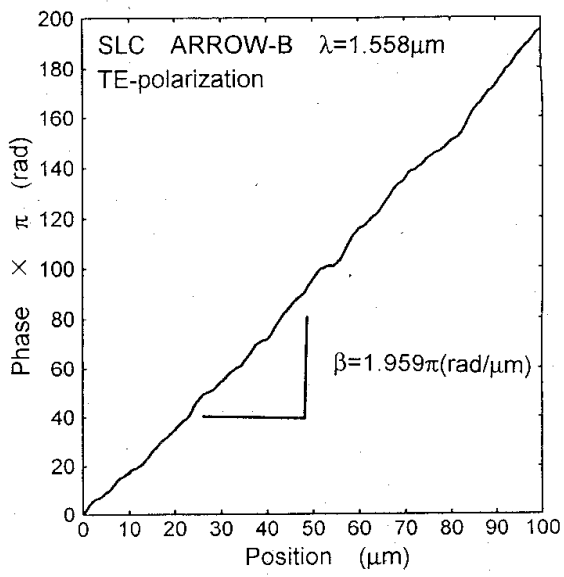

Fig. 5. Measured result of the phase change of the fundamental guided mode in ARROW-B along the waveguide pattern. The theoretical value of the propagation constant is $1.9638 \pi(\mathrm{rad} / \mu \mathrm{m})$.

\section{REFERENCES}

[1] A. Miki, Y. Okamura, and S. Yamamoto, "Optical waveguide directional coupler measurement using a microcomputer assisted TV camera system," J. Lightwave Technol., vol. 7, no. 12, pp. 1912-1918, 1989.

[2] M. Kobayashi, H. Hanafusa, K. Takada, and J. Noda, "Polarizationindependent interferometric optical-time-domain reflectometer," $J$. Lightwave Technol, vol. 9, no. 5, pp. 623-628, 1991.

[3] O. Kamatani and K. Hotate, "Optical coherence domain reflectometry by synthesis of coherence function with nonlinearity compensation in frequency modulation of a laser diode," J. Lightwave Technol., vol. 11, no. 11 , pp. $1854-1862,1993$.

[4] W. V. Sorin and D. M. Baney, "Measurement of Rayleigh backscattering at $1.55 \mu \mathrm{m}$ with $32 \mu \mathrm{m}$ spatial resolution," IEEE Photonics Technol. Lett., vol. 4, no. 4, pp. 374-376, 1992.

[5] A. Kohlhaas, C. Frömchen, and E. Brinkmeyer, "High-resolution OCDR for testing integrated optical waveguides: dispersion-corrupted experimental data corrected by a numerical algorithm," J. Lightwave Technol., vol. 9, no. 11, pp. 1493-1502, 1991.

[6] Y. Kokubun, T. Akiyama, and S. Hosono, "Diagnostics of optical waveguides by heterodyne scattering detection (HSD) method," in 17th European Conf. Optical Commun. 8th Int. Conf. Integrated Optics and Optical Fiber Commun. (ECOC/IOOC'91), Paris, Tu.B5.7, 1991.

[7] Y. Kokubun, T. Kuroiwa, and A. Yamato, "Measurement of birefringence in optical waveguide by heterodyne scattering detection (HSD) method," in 4th Microoptics Conf./11th Gradient-Index Optical Syst. Topical Meeting (MOC/GRIN'93), Kawasaki, C5, 1993.

[8] Y. Toda and M. Ohtsu, "High spatial resolution diagnostics of optical waveguides using a photon-scanning tunneling microscope," Photon. Technol. Lett., vol. 7, no. 1, pp. 84-86, 1995.

[9] T. Pangaribuan, K. Yamada, S. Tiang, H. Ohsawa, and M. Ohtsu, "Reproducible fabrication technique of nanometric tip diameter fiber probe for photon scanning tunneling microscope," Jpn. J. Appl. Phys., Pt. 2, vol. 31, no. 9A, pp. L1302-L1304, 1992. 